\title{
Heart rate response to vasodilator stress: A potential new application for a proven prognostic parameter
}

\author{
Christopher L. Schumann, MD, and Jamieson M. Bourque, MD, MHS ${ }^{a, b}$ \\ a Department of Medicine, University of Virginia Health System, Charlottesville, VA \\ b Department of Radiology, Cardiovascular Imaging Center, University of Virginia Health System, \\ Charlottesville, VA
}

Received Apr 17, 2020; accepted Apr 17, 2020

doi: $10.1007 / \mathrm{s} 12350-020-02168-3$

\section{See related article, pp. 2676-2683}

While the focus of stress testing has traditionally been on the evaluation of electrocardiographic or imaging evidence of ischemia, the hemodynamic response to stress offers a wealth of both diagnostic and prognostic information to the clinician beyond this one application. In particular, heart rate response (HRR) to stress is a well-known predictor of general and cardiac events. A diminished HRR to exercise, termed chronotropic incompetence, is a known predictor of mortality ${ }^{1}$ and coronary artery disease severity. ${ }^{2}$ Even after controlling for left ventricular (LV) function and myocardial ischemia, chronotropic incompetence is a powerful predictor of cardiac events. ${ }^{3}$ Given its prognostic utility, evaluation of HRR should be an integral part of every exercise stress test.

However, the utility of HRR is not limited to exercise testing. For patients undergoing pharmacologic stress, a blunted HRR to vasodilator stress has also been associated with increased mortality. ${ }^{4,5}$ The proposed mechanism by which vasodilators cause an increased heart rate is through direct sympathetic nervous system (SNS) stimulation through $\mathrm{A}(2 \mathrm{~A})$ adenosine receptor activation. ${ }^{6}$ As a result, HRR to vasodilators may provide an indirect assessment of the cardiac SNS, as a

Reprint requests: Jamieson M. Bourque, MD, MHS, Department of Radiology, Cardiovascular Imaging Center, University of Virginia Health System, Box 8006621215 Lee Street, Charlottesville, VA 22908; jbourque@virginia.edu

J Nucl Cardiol 2021;28:2684-6.

1071-3581/ $\$ 34.00$

Copyright (c) 2020 American Society of Nuclear Cardiology. blunted heart rate response could be attributable to autonomic dysfunction or to elevated sympathetic tone in the case of heart failure. ${ }^{7}$

While heart rate response to vasodilators may represent an indirect measure of SNS function, cardiac innervation can be assessed directly through ${ }^{123} \mathrm{I}$ metaiodobenzylguanidine ( $\left.{ }^{123} \mathrm{I}-\mathrm{MIBG}\right)$ imaging. ${ }^{123} \mathrm{I}-$ MIBG is analogous to norepinephrine and its use as a radiotracer allows for the evaluation of abnormalities in cardiac sympathetic innervation. ${ }^{8}$ A large, multicenter trial (ADMIRE-HF) showed that an abnormal ${ }^{123} \mathrm{I}-$ MIBG study, defined as a heart-to-mediastinum (H/M) ratio $<1.6$, had significant prognostic implications in heart failure patients with NYHA class II-III symptoms and LV ejection fraction $(\mathrm{EF})<35 \%$. $^{9}$ Following this study, the FDA approved the use of ${ }^{123}$ I-MIBG in the United States for risk stratification in this population. However, while prognostic value was directly demonstrated, the lack of prospective studies showing the utility of ${ }^{123}$ I-MIBG imaging on treatment decisions in heart failure has limited its reimbursement by health insurance companies. ${ }^{10}$ Currently, the cost of the radiotracer exceeds Centers for Medicare and Medicaid Service (CMS) reimbursement, and third party payers are either offering coverage below the cost of the examination or are not covering the study at all. ${ }^{11}$ As a result, ${ }^{123} \mathrm{I}-\mathrm{MIBG}$ is rarely used in clinical practice.

Given the prognostic benefit of cardiac SNS assessment but challenges in ${ }^{123}$ I-MIBG imaging clinical application, the need for an alternative assessment of cardiac sympathetic function is apparent. In this issue of the Journal, Nappi and colleagues propose that HRR during pharmacologic stress may be an appropriate surrogate. They investigate the relationship between HRR during pharmacological stress testing and myocardial innervation using ${ }^{123} \mathrm{I}-\mathrm{MIBG}$ cardiac 
scintigraphy in 71 patients with known or suspected coronary artery disease. They found a significant correlation between HRR and an abnormal ${ }^{123}$ I-MIBG H/M ratio. On multivariable regression analysis, HRR was a significant predictor of $\mathrm{H} / \mathrm{M}$ ratio.

Although it was significant, the correlation between $\mathrm{HRR}$ and $\mathrm{H} / \mathrm{M}$ ratio was modest $(r=.28)$. For instance, Figure 2 shows that there was substantial scatter in the $\mathrm{HRR}$ in patients who had a $\mathrm{H} / \mathrm{M}$ ratio $<1.6$. However, figure 1 highlights that there is fairly good separation in HRR between those with high and low $\mathrm{H} / \mathrm{M}$ ratio tertiles. Those with a HRR $>20 \%$ appear to have a low likelihood of a low $\mathrm{H} / \mathrm{M}$ ratio $<1.4$ on ${ }^{123} \mathrm{I}-\mathrm{MIBG}$ imaging. Yet the middle tertile, the majority of whom are under the prognostically-significant cutoff of $<1.6$, have a wide variation in HRR. These findings suggest that HRR is not ready to replace ${ }^{123} \mathrm{I}-\mathrm{MIBG}$ imaging for definitive assessment of cardiac sympathetic function, but may be a useful screening tool for autonomic dysfunction, as patients with a low HRR to vasodilator could be considered for further imaging. Still this study represents a promising new application of hemodynamic data routinely obtained during stress testing, and further investigation in larger patient cohorts is warranted to verify its significance. In addition, it is possible that this marker could be combined with other clinical predictors to more reliably identify those with significant cardiac sympathetic dysfunction.

Of particular interest would be a comparison of ${ }^{123} \mathrm{I}$ MIBG uptake and HRR in the heart failure population, as this is where much of the research in cardiac innervation imaging has been concentrated. However, even if HRR is verified as an adequate surrogate for assessing cardiac sympathetic function, there remains the question of whether such as assessment will affect clinical decision making. It is important to note that while large prospective randomized trials have not been completed to verify the utility of cardiac SNS imaging in the management of heart failure patients, there are several promising applications of this imaging modality. One proposed application to heart failure patients would be to assess response to medical therapy. Gerson et al. demonstrated that initiation of beta-blockers leads to improvement in $\mathrm{H} / \mathrm{M}$ ratio in heart failure patients on serial ${ }^{123}$ I-MIBG imaging ${ }^{12}$; furthermore, improvement on ${ }^{123}$ I-MIBG imaging in response to beta-blockers has been shown to be predictive of cardiac events. ${ }^{13}$ Other heart failure medications, including angiotensin receptor blockers ${ }^{14}$ and ACE inhibitors, ${ }^{15}$ have also been shown to improve cardiac sympathetic function. These studies suggest that there may be a role for cardiac SNS imaging in guiding the use of pharmacologic therapy in heart failure patients.
Another promising application of cardiac sympathetic imaging in heart failure patients is to assess the need for an implantable cardioverter-defibrillator (ICD) device. While LVEF $\leq 35 \%$ is used as the principle indication for a primary prevention ICD, a low LVEF has been shown to be an insufficient predictor of ventricular arrhythmias. ${ }^{16}$ In contrast, studies of patients with previously implanted ICDs have found a lower H/ $\mathrm{M}$ ratio on ${ }^{123}$ I-MIBG imaging to be highly predictive of ICD discharges, ${ }^{17,18}$ suggesting that assessment of cardiac innervation can be used to identify patients who would benefit from a primary prevention ICD. A prospective randomized trial was initiated comparing ${ }^{123} \mathrm{I}-\mathrm{MIBG} \mathrm{H} / \mathrm{M}$ ratio-guided ICD implantation to a guidelines-directed approach for patients with NYHA class II-III heart failure and LVEF 25-35\%. Unfortunately, due to slow site/patient recruitment, the study was discontinued in March 2018. ${ }^{11}$ As a result, the question of whether cardiac SNS imaging can predict the need for an ICD remains unanswered.

In summary, there remains much promise in cardiac innervation imaging. However, the limited application of ${ }^{123} \mathrm{I}-\mathrm{MIBG}$ has hindered growth in this field. Identification of a useful surrogate could have significant prognostic and perhaps therapeutic implications for patients with heart failure. Further study is needed to determine if heart rate response to vasodilators is such a surrogate.

\section{Disclosures}

Dr Bourque owns equity in Locus Health and is a consultant for Pfizer and General Electric.

\section{References}

1. Lauer MS, Francis GS, Okin PM, et al. Impaired chronotropic response to exercise stress testing as a predictor of mortality. J Am Med Assoc 1999;281:524-9. https://doi.org/10.1001/jama.281.6. 524.

2. Brener SJ, Pashkow FJ, Harvey SA, et al. Chronotropic response to exercise predicts angiographic severity in patients with suspected or stable coronary artery disease. Am J Cardiol 1995;76:1228-32. https://doi.org/10.1016/s0002-9149(99)803476.

3. Elhendy A, Mahoney DW, Khandheria BK, et al. Prognostic significance of impairment of heart rate response to exercise: Impact of left ventricular function and myocardial ischemia. J Am Coll Cardiol 2003;42:823-30. https://doi.org/10.1016/S0735-1097 (03)00832-5.

4. Bhatheja R, Francis GS, Pothier CE, Lauer MS. Heart rate response during dipyridamole stress as a predictor of mortality in patients with normal myocardial perfusion and normal electrocardiograms. Am J Cardiol 2005;95:1159-64. https://doi.org/10. 1016/j.amjcard.2005.01.042. 
5. Abidov A, Hachamovitch R, Hayes SW, et al. Prognostic impact of hemodynamic response to adenosine in patients older than age 55 years undergoing vasodilator stress myocardial perfusion study. Circulation 2003;107:2894-9. https://doi.org/10.1161/01.CIR.000 0072770.27332 .75 .

6. Dhalla AK, Wong M-Y, Wang W-Q, et al. Tachycardia caused by A2A adenosine receptor agonists is mediated by direct sympathoexcitation in awake rats. J Pharmacol Exp Ther 2006;316:695702. https://doi.org/10.1124/jpet.105.095323.

7. Gorur GD, Ciftci EA, Kozdag G, et al. Reduced heart rate response to dipyridamole in patients undergoing myocardial perfusion SPECT. Ann Nucl Med 2012;26:609-15. https://doi.org/10. 1007/s12149-012-0618-z.

8. Chirumamilla A, Travin MI. Cardiac applications of 123I-mIBG imaging. Semin Nucl Med 2011;41:374-87.

9. Jacobson AF, Senior R, Cerqueira MD, et al. Myocardial iodine123 meta-iodobenzylguanidine imaging and cardiac events in heart failure. Results of the prospective ADMIRE-HF (AdreView Myocardial Imaging for Risk Evaluation in Heart Failure) study. J Am Coll Cardiol 2010;55:2212-21. https://doi.org/10.1016/j.jacc. 2010.01.014.

10. Scholte AJHA. The future of cardiac 123-I MIBG imaging. Eur J Nucl Med Mol Imaging 2016;43:2381-2.

11. Travin MI, Matsunari I, Thomas GS, et al. How do we establish cardiac sympathetic nervous system imaging with 123I-mIBG in clinical practice? Perspectives and lessons from Japan and the US. J Nucl Cardiol 2019;26:1434-51. https://doi.org/10.1007/s12350018-1394-5.

12. Gerson MC, Craft LL, Mcguire N, et al (2002) Carvedilol improves left ventricular function in heart failure patients with idiopathic dilated cardiomyopathy and a wide range of sympathetic nervous system function as measured by iodine 123 metaiodobenzylguanidine. https://doi.org/10.1067/mnc.2002. 127717
13. Fujimoto S, Inoue A, Hisatake S, et al. Usefulness of 123Imetaiodobenzylguanidine myocardial scintigraphy for predicting the effectiveness of $\beta$-blockers in patients with dilated cardiomyopathy from the standpoint of long-term prognosis. Eur J Nucl Med Mol Imaging 2004;31:1356-61. https://doi.org/10.1007/s002 59-004-1557-2.

14. Kasama S, Toyama T, Kumakura H, et al. Effects of candesartan on cardiac sympathetic nerve activity in patients with congestive heart failure and preserved left ventricular ejection fraction. J Am Coll Cardiol 2005;45:661-7. https://doi.org/10.1016/j.jacc.2004.1 1.038 .

15. Somsen GA, Van Vlies B, De Milliano PAR, et al. Increased myocardial [123I]-metaiodobenzylguanidine uptake after enalapril treatment in patients with chronic heart failure. Heart 1996;76:218-22. https://doi.org/10.1136/hrt.76.3.218.

16. Buxton AE, Lee KL, Hafley GE, et al. Limitations of ejection fraction for prediction of sudden death risk in patients with coronary artery disease: Lessons from the MUSTT study. J Am Coll Cardiol 2007;50:1150-7. https://doi.org/10.1016/j.jacc.2007. 04.095.

17. Arora R, Ferrick KJ, Nakata T, et al. I-123 MIBG imaging and heart rate variability analysis to predict the need for an implantable cardioverter defribillator. J Nucl Cardiol 2003;10:12131. https://doi.org/10.1067/mnc.2003.2.

18. Nagahara D, Nakata T, Hashimoto A, et al. Predicting the need for an implantable cardioverter defibrillator using cardiac metaiodobenzylguanidine activity together with plasma natriuretic peptide concentration or left ventricular function. J Nucl Med 2008;49:225-33. https://doi.org/10.2967/jnumed.107.042564.

Publisher's Note Springer Nature remains neutral with regard to jurisdictional claims in published maps and institutional affiliations. 